\title{
Synaptic Dynamics Influence the Phase of a Neural Response
}

\author{
Bruce P. Graham \\ Computing Science and Mathematics, School of Natural Sciences, University of Stirling \\ Stirling, Scotland FK9 4LA, U.K. \\ b.graham@cs.stir.ac.uk
}

\begin{abstract}
The postsynaptic response of a neuron to timevarying inputs is determined by the interaction of presynaptic spike times with the short-term dynamics of each synapse. Such synaptic dynamics makes the postsynaptic neuron more sensitive to input rate fluctuations than to average firing rates. Here we show that the postsynaptic neural spiking response to a rhythmically frequency-modulated population input can exhibit a predictive phase lead due to depressing synapses. The magnitude of the lead increases with increasing correlations between the input spike trains. Facilitation and vesicle recycling rates also influence the phase.
\end{abstract}

\section{INTRODUCTION}

Most chemical synapses between neurons exhibit a dynamic response on millisecond time scales to arriving inputs from the presynaptic neuron [1]. Such dynamics, that include depression and facilitation, are know as synaptic short term plasticity (STP), to distinguish them from the long term changes in synaptic strength thought to underlie learning. STP can have a significant effect on the ability of a synaptic pathway to transmit information about the steady-state input signal rate. Synaptic depression essentially eliminates rate information at high stimulus rates, as the mean synaptic current becomes independent of frequency due to the increased arrival rate of input spikes being balanced by a corresponding decrease in the mean unitary EPSC amplitude because of depression [2],[3]. However, changes in stimulus rate do induce a transient postsynaptic response. At the opposite extreme, STP can result in synaptic pathways transmitting significant information about presynaptic interspike intervals, with this information being carried by the per-spike EPSC amplitudes [4],[5] and being evident in the output spiking response [6]. STP ensures that EPSC amplitude is a strong function of the ISI over a significant operating range of intervals [7]. In between these two extremes, neural systems may deal with relatively slowly time varying signals carried by the combined spiking of populations of cells. Rhythmic behaviour in motor systems, for example, can involve oscillatory signals on the order of $1 \mathrm{~Hz}$. The time course of such signals is the same order of magnitude of many of the time constants of different STP mechanisms, such as recovery from depression and facilitation. As a consequence, the amplitude and phase of a rhythmically varying signal can be strongly affected by the STP of the synaptic pathways the signal is transmitted by.
In this paper we consider two aspects of STP that have largely not been explored. We examine how STP affects the phase of the spiking output response of neurons driven by a sinusoidally frequency-modulated input signal. The input signal is encoded as the firing rates of input neurons forming synapses with an output neuron. Different combinations of STP mechanisms at each synapse are explored. In addition, we consider also the effect of the configuration of the synaptic pathway onto an output neuron. This pathway is assumed to consist of a fixed number of release sites that are divided between active zones, with each active zone being the presynaptic axonal target of a single input neuron. For the same number of release sites, at one extreme a configuration consists of the output neuron receiving input from a large number of neurons through independent active zones, each containing a single release site. At the other extreme, the output neuron is driven by a single input neuron through a giant synapse containing a single active zone with a very large number of release sites. Such differences in synaptic configuration do affect how STP determines the output neuron response [8],[9]. As will be shown here, the interaction between STP and synaptic configuration has a significant effect of the phase of the output response to rhythmically varying inputs.

\section{MethodS}

We use computer simulations of a simple spiking neuron driven by frequency-modulated, Poisson-distributed spike trains arriving at dynamic synapses. How the output spiking response compares to the rhythmically varying input is examined.

\section{A. Vesicle Release Model}

To model dynamic synapses we use a simple model of vesicle availability and release at individual synaptic release sites [3],[8],[10]. The available pool of readily-releasable vesicles (RRVP) is depleted by stochastic vesicle release on arrival of a presynaptic action potential. The RRVP recovers by replenishment from an infinitely large reserve pool. The rate of recovery may be an increasing function of presynaptic stimulation frequency. The probability of vesicle release, $p_{v}$, is also subject to facilitation so that it increases with stimulation frequency.

A single release site may either be occupied by a single releasable vesicle, or it may be empty. For a sequence of 
presynaptic spikes arriving at times $t_{\mathrm{s}}$, the average release site occupancy, $n$, is given by:

$$
\frac{d n}{d t}=\frac{1-n}{\tau_{\text {rec }}}-\sum_{s} p_{v} n \delta\left(t-t_{s}\right)
$$

The recovery time may be frequency-dependent, decreasing by a fraction, $\Delta \tau_{r}$, with each presynaptic spike:

$$
\frac{d \tau_{r e c}}{d t}=\frac{\tau_{r 0}-\tau_{r e c}}{\tau_{r F D R}}-\sum_{s} \Delta \tau_{r} \tau_{r e c} \delta\left(t-t_{s}\right) .
$$

The vesicle release probability facilitates by a fraction, $\Delta p_{f}$, on the arrival of each presynaptic spike:

$$
\frac{d p_{v}}{d t}=\frac{p_{b}-p_{v}}{\tau_{f}}+\sum_{s} \Delta p_{f} \cdot\left(1-p_{v}\right) \delta\left(t-t_{s}\right) \text {. }
$$

The amplitude of the postsynaptic current response, PSR, is determined by the amount of release and the quantal size, $q$ :

$$
P S R=q p_{v} n \text {. }
$$

This average model of a release site is implemented in the form of a discrete-time, stochastic, finite-state automaton. At a particular time point, a release site may be occupied by a releasable vesicle, or may be empty. An occupied site may release its vesicle with probability $p_{v}$ on arrival of a presynaptic spike. An empty site may be refilled during the small simulation time interval $\Delta t$, with probability $\Delta t / \tau_{\text {rec }}$.

\section{B. Postsynaptic Cell}

The postsynaptic cell is modelled as a single cylindrical compartment, with length and diameter of $20 \mu \mathrm{m}$. The membrane generates fast sodium and delayed-rectifier potassium currents, resulting in action potentials. These are modelled using the standard Hodgkin-Huxley formalism.

Each presynaptic release site corresponds to a postsynaptic specialisation containing a population of AMPA channels. Release of a vesicle generates an excitatory postsynaptic current (EPSC) with a rise time of 0.1 msecs, fall time of $1 \mathrm{msec}$ and reversal potential of $0 \mathrm{mV}$. The peak synaptic conductance is determined by the quantal size, $q$.

\section{Synaptic Configuration}

An axon from a presynaptic cell is assumed to make contact with the postsynaptic cell via a single active zone (AZ) that contains a specified number of release sites. A synaptic pathway will consist of a set of AZs, each driven by a different presynaptic cell.

Different synaptic configurations are constructed from a fixed total number of release sites, $\mathrm{N}=512$. The release sites are equally subdivided between a specified number of AZs (Fig. 1). Thus configurations range from a single AZ containing 512 release sites, of the form of giant synapses such as the calyx of Held [11], to 512 AZs, each containing a single release site, of the form of excitatory synaptic input onto cortical pyramidal cells from a population of presynaptic cells whose activity encodes a common stimulus [2].

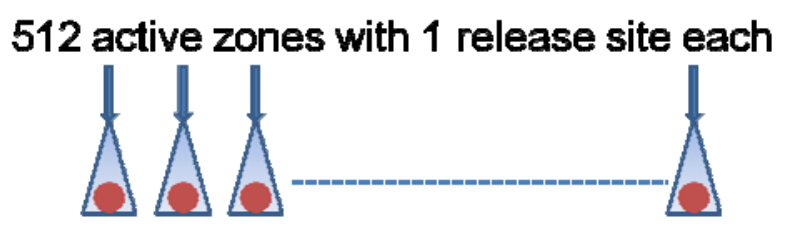

128 active zones with 4 release sites each

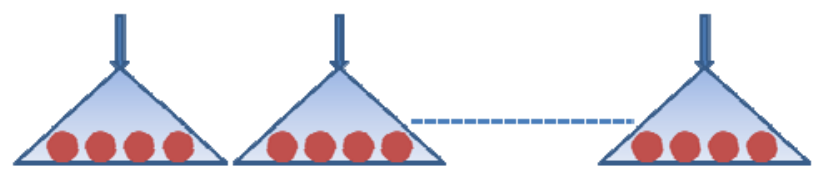

1 active zone with 512 release sites

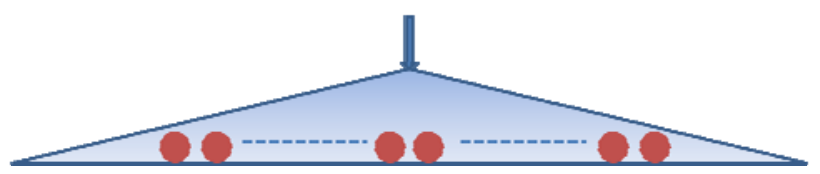

Fig. 1. Examples of different synaptic configurations. 512 vesicle release sites are equally distributed between a given number of active zones. Each active zone is the target of a single presynaptic axon (arrows) being driven by a unique input neuron (not shown).

\section{Input Stimulus}

The input signal is a $30 \mathrm{~Hz}$ carrier frequency that is modulated sinusoidally between $50 \mathrm{~Hz}$ and $10 \mathrm{~Hz}$ at different modulation frequencies $(0.1,1$ and $3 \mathrm{~Hz})$. Input spike trains are generated as independent, inhomogeneous Poisson processes. Spike times in individual trains were produced by the thinning method for generating inhomogeneous Poisson series. Spike trains to different active zones have the same time-varying mean frequency but individual spikes are uncorrelated in time. Thus the "cortical" configuration is a completely uncorrelated pathway in which all 512 release sites are driven by an independent presynaptic spike train. In contrast, the "giant" configuration is a completely correlated pathway in which all 512 releasable sites may release independently, but are driven by spikes from a single presynaptic cell.

\section{E. Simulations}

Different combinations of release site dynamics are considered: (1) depression-only (D); (2) depression plus facilitation (D+F); and (3) depression plus facilitation and frequency-dependent recovery $(\mathrm{D}+\mathrm{F}+\mathrm{R})$. In the results shown here, the release site parameters are: $\tau_{r 0}=500 \mathrm{msecs}$, $\tau_{r F D R}=500 \mathrm{msecs}, \Delta \tau_{r}=0.2, p_{b}=0.25, \tau_{f}=500 \mathrm{msecs}, \Delta p_{f}=0.1 . \Delta \tau_{r}$ and $\Delta p_{f}$ are set to 0 when $\mathrm{F}$ and $\mathrm{R}$ are not required.

Simulation runs with particular synaptic configurations are repeated 8 times with the same input streams, but with different random seeds, resulting in different vesicle release patterns. Where possible, runs are also repeated with completely different sets of input streams to the same synaptic configuration. Simulations start with 1 second of stimulation 
at $30 \mathrm{~Hz}$ before the sinusoidal modulation begins. Modulation continues for at least 5 cycles.

Postsynaptic cell spike times are collected and collated from all runs with a particular synaptic configuration. Spike time histograms are produced by binning in $5 \mathrm{msec}$ time bins. Sine waves corresponding to the underlying input carrier signal are optimally fit to the histograms via least squares minimisation. The phase of a response is taken to be the phase of the fitted sine wave, relative to a phase of 0 for the input signal.

\section{RESULTS}

Initial simulations considered the postsynaptic cell spiking response when driven by a $1 \mathrm{~Hz}$-modulated carrier signal through different synaptic configurations, with particular synaptic dynamics. Fig. 2 shows raster plots of spiking output from distinct postsynaptic cells driven through depressingonly synapses (no facilitation or frequency-dependent recovery). By inspection it can be seen that the peak spiking response via the giant synapse configuration $(A Z=1)$ is out of phase with the input modulation, whereas for synaptic configurations with more, but smaller active zones ( $A Z=4$ and $\mathrm{AZ}=32$ ) the response is increasingly in phase.

This phase relationship is clearer in Fig. 3. Here, spike times are binned and plotted as histograms, both for the input spike streams and for the postsynaptic cell responses. A $1 \mathrm{~Hz}$ sinusoid was fitted to these histograms via a least-squares fitting procedure optimising over amplitude and phase. The relative phases are easily seen when these sinusoids are overlayed (Fig. 3e).

The smallest sized active zone configuration shown $(A Z=32)$ essentially follows the input modulation, but with a small phase lead of 17 degrees. The giant synapse $(A Z=1)$ exhibits an essentially out-of-phase response (phase lead of 104 degrees) that is somewhat asymmetric with respect to the sinusoidal input, with large, narrow peaks in firing. With a few active zones $(A Z=4)$ there is an intermediate phase lead of 56 degrees. The smallest phase lead is 14 degrees when there are 512 active zones, each with a single release site (not shown in Fig. 3).

The explanation for these results is as follows. For a purely depressing synapse, the mean release probability at an individual release site is out of phase with the input signal frequency modulation, since that it decreases as the signal frequency increases, and vice versa. The mean probability is approximately inversely proportional to the driving frequency at high frequencies [3]. As a consequence, in the uncorrelated "cortical" configuration of 512 independent AZs, the mean synaptic current is proportional to the driving frequency multiplied by the release probability, and so is only shallowly dependent on frequency at high frequencies. For the frequencies used here, the depression at $50 \mathrm{~Hz}$ is not quite 5 times the depression at $10 \mathrm{~Hz}$, allowing the synaptic current to following the modulated driving frequency. However, the finite time constant of depression results in a small phase lead in the resultant output spiking response as depression increases as the driving frequency increases, leading to the summed synaptic current peaking before the peak of the driving frequency.
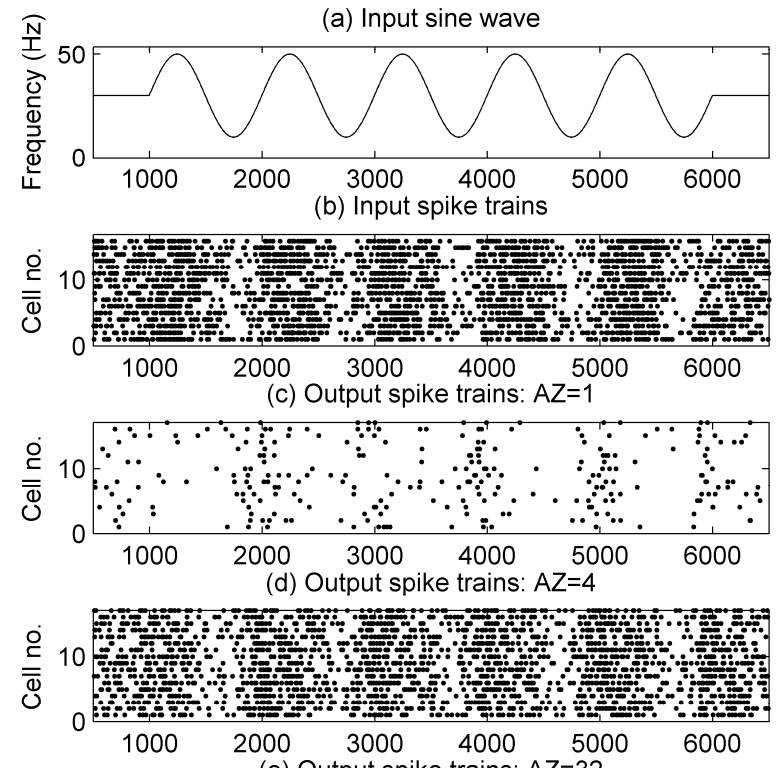

(e) Output spike trains: $A Z=32$

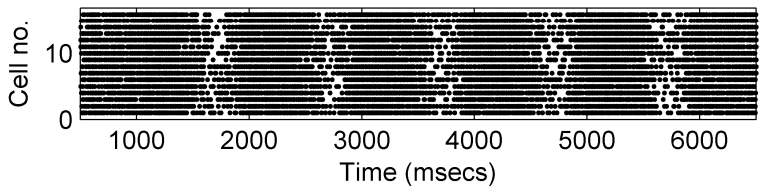

Fig. 2. Raster plots of postsynaptic spiking in response to $1 \mathrm{~Hz}$-modulated population inputs for different synaptic configurations with depressing synapses. Each postsynaptic cell was driven by a different set of input spike trains with identical frequency characteristics (single runs with each cell).

The situation is quite different in the correlated "giant" synapse configuration. In this case there is no temporal summation of EPSPs across release sites. Instead, the population of sites releases synchronously on arrival of each presynaptic action potential. The amplitude of the consequent EPSC is thus directly proportional to the vesicle release probability, which, in turn, is an inverse function of the input frequency. In the fluctuation-driven spiking regime used here, as the input frequency increases, EPSCs depress to the point at which a population EPSC no longer drives the output to spiking threshold. On the other hand, as the input frequency decreases, output spiking becomes more reliable as the population EPSC amplitude increases. The spike histogram obtained over a population of cells does not exhibit a perfectly out-of-phase response, compared to the input signal, since the most reliable spiking corresponds to the lowest input frequency. Thus the peak of the histogram is shifted to when the input frequency begins to increase, so that there are both more input spikes per unit time and depression is still building up. Strictly speaking, the population response shown in Fig. 3b exhibits a very large phase lag, since the output cells do not respond at all to the initial peak in input frequency, with the first large response occurring on the upward slope of the second input modulation cycle. However, given that this 
response is signalling the start of this new cycle, for continuous modulation it seems more appropriate to deem this a functional phase lead.

(a) Input spike histogram
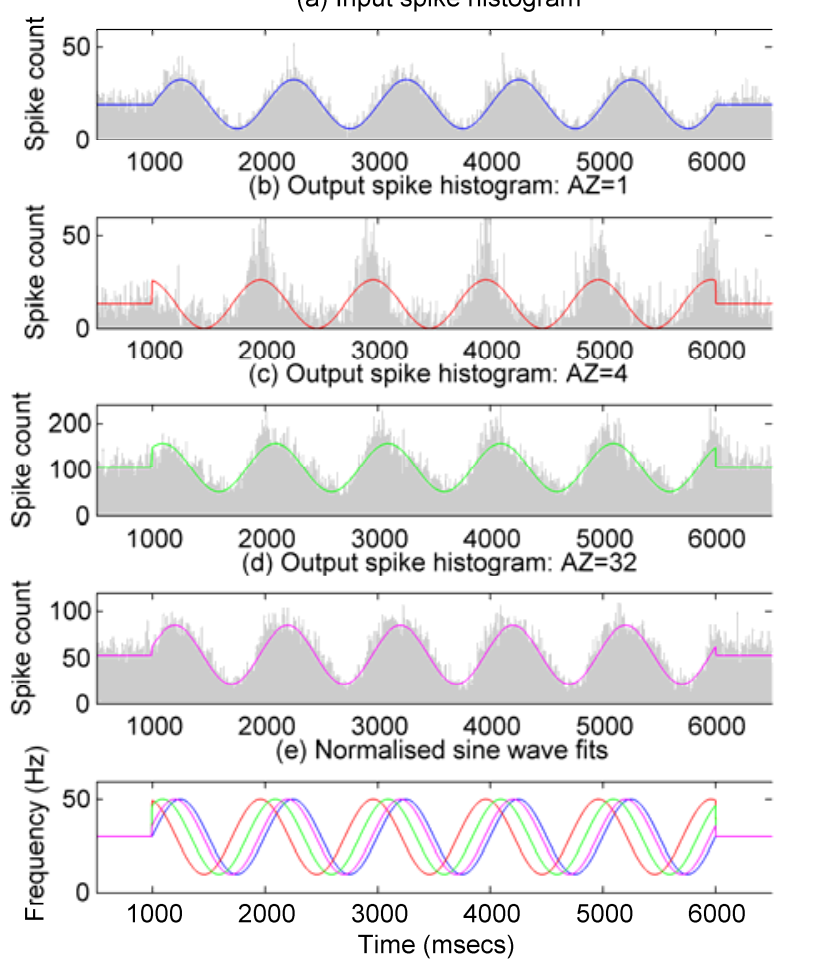

Fig. 3. Histograms of postsynaptic spiking in response to $1 \mathrm{~Hz}-$ modulated population inputs for different synaptic configurations with depressing synapses. Spike times from all cells in all runs with a particular synaptic configuration are binned in 5 msec time bins. Overlayed lines are optimally fitted sine waves. Panel (e) shows all fitted sine waves with normalised amplitudes (left to right for phase: $\mathrm{AZ}=1, \mathrm{AZ}=4, \mathrm{AZ}=32$, Inputs).

For synaptic configurations between the two extremes, the response shifts smoothly, but quite rapidly from the spatial summation of the giant synapse to the temporal summation of the cortical synapse. Thus the phase lead reduces quickly as the number of active zones driven by independent spike trains increases (Fig. 4a). So a configuration even with only 4 AZs is a mixture of spatial and temporal summation and has a phase lead intermediate between the extremes. The lead is almost at the extreme cortical level with 32 AZs.

The phase of the output spiking response for different synaptic configurations and STP mechanisms is summarised in Fig. 4. Facilitation (F) in combination with depression (D) increases the phase lead at correlated synapses (few AZs), but decreases it for uncorrelated configurations (many AZs). Frequency-dependent release site recovery $(\mathrm{R})$ results in an inphase response, similar to that obtained with static synapses. However, facilitation in combination with frequencydependent recovery results in a large phase lag for correlated synapses. This is further illustrated and explained below. (a)

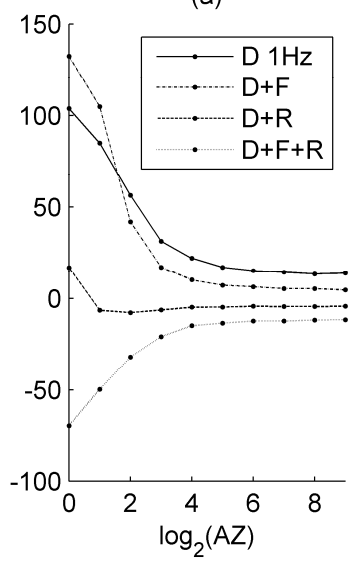

(b)

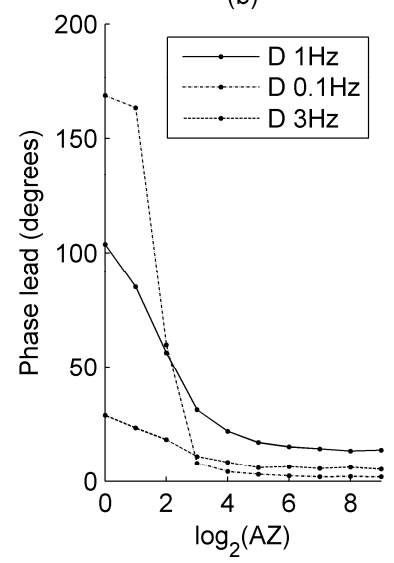

Fig. 4. Phase of response to rhythmic population inputs for different synaptic configurations and modulation frequencies. (a) Modulation at $1 \mathrm{~Hz}$ for different combinations of synaptic dynamics. D: depression; D+F: depression + facilitation; $\mathrm{D}+\mathrm{F}+\mathrm{R}$ : depression + facilitation + frequency-dependent recovery. (b) Various modulation frequencies with a depressing synapse.

Note that a static synapse (results not shown), in which all release sites always have a vesicle available for release with a fixed probability, generates an in-phase postsynaptic response regardless of synaptic configuration. These responses have a very small phase lag of around 2 degrees or less, due to the delay between vesicle release and postsynaptic action potential generation.

At all modulation frequencies $(0.1,1,3 \mathrm{~Hz})$ and in all configurations, synaptic depression results in the output response leading the input oscillations (Fig. 4b). The lead increases as the inputs become more correlated (decreasing AZ). The effect is amplified by facilitation for correlated synapses $(A Z=1$ or 2 ), but reduced for less correlated pathways. Also, the lead decreases with increasing modulation frequency for correlated synapses, but is maximal at $1 \mathrm{~Hz}$ for uncorrelated pathways. Clearly, correlated synapses (few AZs) are much more sensitive to STP mechanisms and the modulation frequency than uncorrelated synapses (many AZs).

The effects of different release site dynamics for the correlated "giant" synapse $(\mathrm{AZ}=1)$ are illustrated in Fig. 5. Facilitation results in earlier spiking as the input frequency first falls then begins to increase, giving an even greater phase lead than for the depressing-only synapse. Frequencydependent recovery is able to sufficiently speed release site refilling that depression on the rising $1 \mathrm{~Hz}$ modulation cycle to $50 \mathrm{~Hz}$ is reduced, but not eliminated. Across the frequency modulation, a reasonably flat spiking response results and any encoding of the modulation is essentially lost from the output spiking response. With added facilitation, output spiking is boosted on the falling phase of the input modulation, when input spiking is still reasonably fast and release site refilling has been speeded to sites that now have an increased release probability. This results in an output that encodes in the input modulation, but with a significant phase lag. 
(a) Input spike histogram

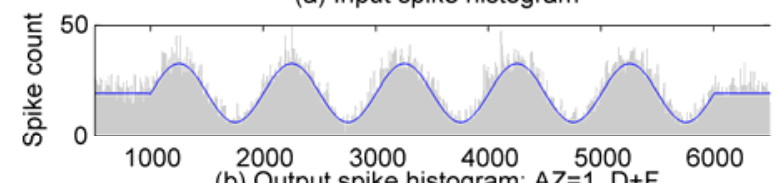

(b) Output spike histogram: $A Z=1, D+F$

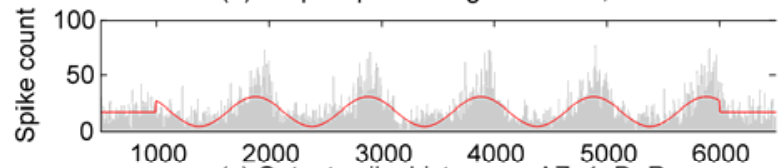

(c) Output spike histogram: $A Z=1, D+R$

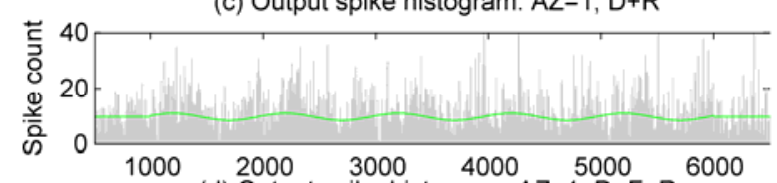

(d) Output spike histogram: $A Z=1, D+F+R$
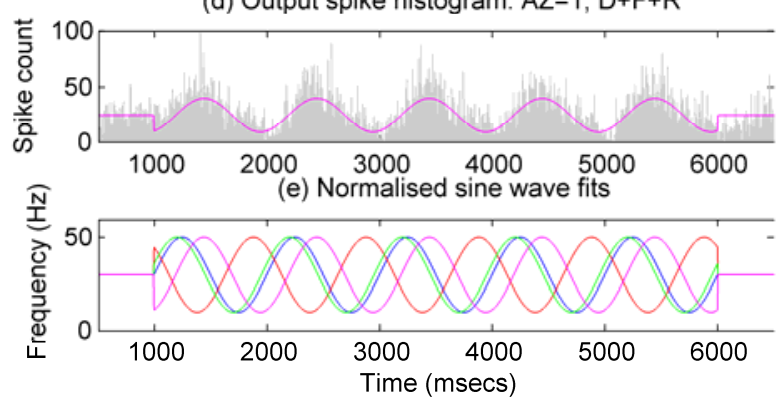

Fig. 5. Histograms of postsynaptic spiking in response to $1 \mathrm{~Hz}$-modulated population inputs for the giant synapse $(A Z=1)$ with different release site dynamics. Overlayed lines are optimally fitted sine waves. Panel (e) shows overlay of fitted sine waves with normalised amplitudes (left to right for phase: $\mathrm{D}+\mathrm{F}, \mathrm{D}+\mathrm{R}$, input, $\mathrm{D}+\mathrm{F}+\mathrm{R})$.

The effect of different release site dynamics on the cortical configuration $(\mathrm{AZ}=512)$ is somewhat similar, but not identical (not shown). Now facilitation helps sustain firing for longer as the input frequency increases, resulting in a reduction in the phase lead of the output. The reduction in depression at high frequencies with frequency-dependent recovery now results in output spiking closely following the input modulation but with a small phase lag, as does a purely static synapse. The addition of facilitation increases the phase lag a little, as again spiking is boosted on the falling phase of the oscillation.

The synaptic time constants mean that the output spiking response varies with modulation frequency, as this interacts with the time course of depression and facilitation. Fig. 6 shows the response of the giant synapse $(A Z=1)$ with depressing synapses to modulations of $0.1,1$ and $3 \mathrm{~Hz}$.

For very slow modulation $(0.1 \mathrm{~Hz})$, the output response is nearly completely out of phase with the input. With such a slow modulation, depression and recovery have time to follow the input frequency. As the frequency increases, depression drives the EPSC amplitude below the spiking threshold for the postsynaptic cell. Conversely, recovery from depression as the input frequency decreases increases the EPSC amplitude and the output cell spikes in correspondence with the lowest input frequencies. (a) $A Z=1,0.1 \mathrm{~Hz}$

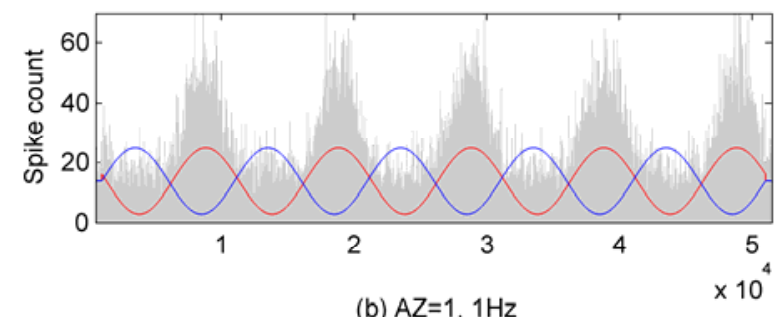

(b) $A Z=1,1 \mathrm{~Hz}$

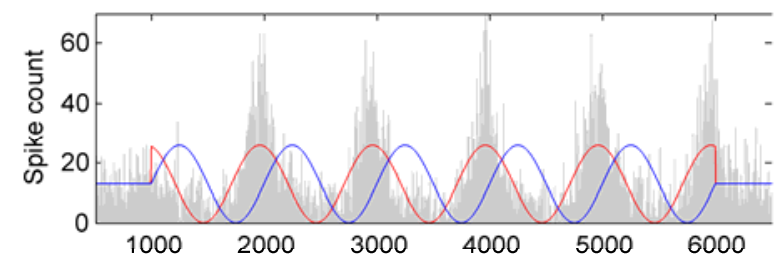

(c) $A Z=1,3 \mathrm{~Hz}$

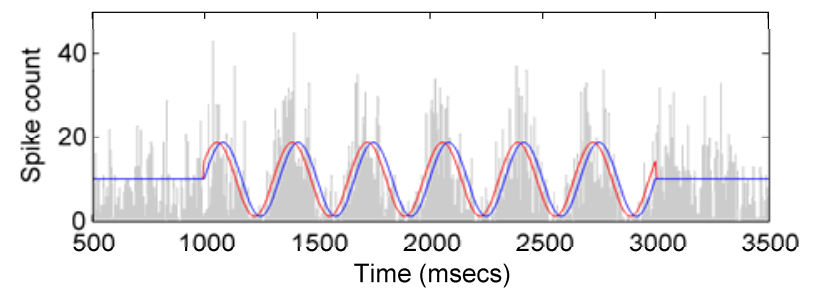

Fig. 6. Histograms of postsynaptic spiking in response to population inputs sinusoidally-modulated at $0.1,1$ or $3 \mathrm{~Hz}$, for the giant synapse $(\mathrm{AZ}=1)$ with depression. Overlayed lines in phase with the histogram peaks are optimally fitted sine waves. Out-of-phase overlayed lines show the input sine wave.

At higher frequencies, output spiking occurs on the rising input phase before depression has a chance to progress. This is accentuated with input modulation frequency, so that at $3 \mathrm{~Hz}$ the output spiking exhibits a true, but small phase lead. The output cell increases its spike rate even on the first rising cycle, unlike at $0.1 \mathrm{or} 1 \mathrm{~Hz}$.

With the cortical configuration $(\mathrm{AZ}=512)$ the output response exhibits a small phase lead at all frequencies (see Fig. $3 \mathrm{~b})$, however the largest lead is at $1 \mathrm{~Hz}$. At $0.1 \mathrm{~Hz}$, the depression level follows the input frequency. It is maximal at $50 \mathrm{~Hz}$, but this is still insufficient to eliminate an increase in output firing with the increase in driving frequency. Thus the output response essentially tracks the input. At $3 \mathrm{~Hz}$ modulation, depression is little modulated by changes in the input frequency, again resulting in the output largely tracking the input. At $1 \mathrm{~Hz}$, depression has time to increase as the firing frequency increases, but the increase in the input frequency is sufficiently fast that the onset response before the synapses depress dominates the output spiking, resulting in a significant phase lead (but still small compared to the giant synapse configuration).

\section{CONCLUSION}

We have shown that synaptic short term plasticity (STP) can significantly affect the phase of the neural output spiking response to rhythmically varying inputs, when the dominant 
time constants of STP are of the same order as the frequency of input variation. For depressing synapses, the output response typically exhibits a phase lead as a result of a strong transient response to changes in input frequency. The magnitude of this lead is very different, depending on the configuration of the synaptic pathway, which determines the spatio-temporal integration of the postsynaptic signal.

A relatively small, but potentially significant phase lead is present in a "cortical” pathway consisting of a large number of small active zones, each driven by a different input cell, with all input cells firing at the same mean frequency, but with independent Poisson-distributed firing times. Temporal summation of EPSCs is significant in this configuration, leading to the mean postsynaptic current becoming largely independent of frequency in the steady state for high input frequencies. In this situation, the output firing is dominated by the transient synaptic response to a change in input frequency, before a new steady state depressed level is reached.

On the other hand, the postsynaptic response due to a giant synapse, containing a single active zone with a large number of release sites, is dominated by spatial summation of the unitary EPSCs from each release site on arrival of a presynaptic spike, with minimal summation of population EPSCs from successive spikes. Here the firing threshold of the postsynaptic cell is set such that the population EPSC depresses below this threshold at the highest input frequencies, but is well above threshold at the lowest frequencies. This results in a very strongly out-of-phase response of the output neuron.

In the simulated synaptic pathways demonstrated here, there is a sharp transition from the spatial-summationdominated to the temporal-summation-dominated regime, which takes place when the number of active zones reaches from 8 to 16 (out of a possible 512; see Fig. 4). Giant synapses, such as the end bulb of Held and the calyx of Held [11] in the mammalian auditory brainstem, definitely fall into the spatial summation class. Such synapses also exhibit significant STP. However, the obvious hypothesis is that these synapses are designed to be highly reliable in generating postsynaptic spiking, even at high input frequencies. Experimental recordings from these postsynaptic cells show that this is likely to be true, though postsynaptic responses may not follow perfectly the input spike train [12]. This indicates that the postsynaptic firing threshold is usually below the level of even highly depressed EPSCs. Thus the large phase shifts seen here, and the effects of STP in general, are not strongly evident at these synapses, though they could be revealed in the right conditions. This leaves the question as to whether STP at these synapses is simply an artefact of chemical synaptic transmission that has no functional consequence. In vivo postsynaptic responses at the calyx of Held do, however, exhibit stronger phase locking to low frequency input modulations than the spike-train-encoded input, reminiscent of the narrowly peaked spike histograms seen in the simulations (Fig. 3b; compare with Fig. 12 of [12]).

Most synaptic pathways in the cortex fall within the temporal summation class. Even in the extreme case of a very large number of asynchronously-driven independent small active zones, a significant phase lead arises at particular frequencies of input modulation. For a $1 \mathrm{~Hz}$ modulation, this lead is around 14 degrees, or equivalently about 40 msecs. Such shifts have been seen in a similar model with depressing synapses in which the inputs are derived as the population spiking response to visual stimuli of sinusoidally varying gratings [13]. This lead is well in excess of transmission delays for which it might compensate. It could contribute to inhibition leading excitation in visual cortical responses to light flashes [14]. Inhibitory interneurons are typically driven by multiple contacts from afferent excitatory cells, and so could exhibit a larger phase lead than in excitatory-toexcitatory cell pathways. This could compensate for the extra delay inherent in the disynaptic inhibitory pathway feeding inhibition onto excitatory cells.

The vestibulo-ocular reflex is a circuit driven by stimuli varying at Hertz. This circuit must maintain a gain of one, such that eye movements can exactly compensate for head movements, allowing an animal to maintain gaze on a fixed target. Significantly, recent data indicate that synapses in the vestibular nuclei do not exhibit STP [15]. We would suggest that this is necessary precisely to avoid the effects demonstrated in our simulations.

\section{REFERENCES}

[1] R Zucker, WG Regehr: Short-term synaptic plasticity. Nature 431:796803, 2004.

[2] LF Abbott, JA Varela, K Sen, SB Nelson: Synaptic depression and cortical gain control. Science 275:220-224, 1997.

[3] M Tsodyks, H Markram: The neural code between neocortical pyramidal neurons depends on neurotransmitter release probability. Proc Natl Acad Sci. USA 94:719-723, 1997.

[4] G Fuhrmann, I Segev, H Markram, M Tsodyks: Coding of temporal information by activity dependent synapses. J Neurophysiol 87:140148, 2002.

[5] M London, A Schreibman, M Hausser, E Matthew, I Segev: The information efficacy of a synapse. Nature Neurosci 5:332-340, 2002.

[6] B Lindner, D Gangloff, A Longtin, JE Lewis: Broadband coding with dynamic synapses. J Neurosci 29:2076-2088, 2009.

[7] Z Yang, M Hennig, M Postlethwaite, ID Forsythe, BP Graham: Wideband information transmission at the calyx of Held. Neural Computation 21:991-1017, 2009.

[8] BP Graham, C Stricker: Short term plasticity provides temporal filtering at chemical synapses. LNCS, 5164:268-276, Springer, 2008.

[9] J de la Rocha, N Parga: Short-term synaptic depression causes a nonmonotonic response to correlated stimuli. J Neurosci 25:8416-8431, 2005.

[10] G Fuhrmann, AI Cowan, I Segev, MV Tsodyks, C Stricker: Multiple mechanisms govern the dynamics of depression at neocortical synapses of young rats. J Physiol 557:415-438, 2004.

[11] R Schneggenburger, ID Forsythe: The calyx of Held. Cell Tissue Res 326:311-337, 2006.

[12] C Kopp-Scheinpflug, WR Lippe, GJ Dorrscheidt, R Rubsamen: The medial nucleus of the trapezoid body in the gerbil is more than a relay: comparison of pre- and postsynaptic activity. JARO 4:1-23, 2002.

[13] FS Chance, SB Nelson, LF Abbott: Synaptic depression and the temporal response characteristics of V1 cells. J Neurosci 18:4785-4799, 1998.

[14] JG Mancilla, M Fowler, PS Ulinski: Responses of regular spiking and fast spiking cells in turtle visual cortex to light flashes. Vis Neurosci 15:979-993, 1998. 